\title{
Avaliação da morfologia e propriedades mecânícas de compósitos laminados a base de epóxi, cortiça e microesferas de vidro
}

\author{
Evaluation of the morphology and mechanical \\ properties of laminated composites based on \\ epoxy, cork and glass microspheres
}

\author{
Bruno Santos Pereira ${ }^{1}$, Renata Barbosa ${ }^{2}$, \\ Tatianny Soares Alves ${ }^{2}$
}

\footnotetext{
${ }^{1}$ Programa de Pós-Graduação em Engenharia de Materiais, Instituto Federal do Piauí, 64000-040, Teresina, Piauí, Brasil.

${ }^{2}$ Curso de Engenharia de Materiais e Programa de Pós-Graduação em Ciência e Engenharia dos Materiais, Universidade Federal do Piauí, 64049-550, Teresina, Piauí, Brasil.

e-mail: bruno_multi@hotmail.com,rrenatabarbosa@yahoo.com, tsaeng3@yahoo.com.br
}

\section{RESUMO}

Neste trabalho foram desenvolvidos compósitos poliméricos epoxídicos laminados a base de cortiça e microesferas ocas de vidro. Os compósitos laminados foram confeccionados manualmente e avaliados quanto às características morfológicas em microscópio eletrônico de varredura e a influência da incorporação das cargas foi avaliada por meio da densidade e da resistência à flexão. De um modo geral, foi observada boa dispersão tanto do pó de cortiça como das microesferas ocas de vidro nas camadas centrais do laminado, porém os núcleos a base de cortiça apresentaram melhor adesão à matriz. Foi observada ainda a redução na densidade de todos os compósitos com relação à matriz pura e que os módulos de flexão dos compósitos a base de cortiça e os com microesferas ocas de vidro, mesmo com valores próximos, foram superiores ao módulo de flexão do sistema epóxi puro. Compósitos laminados com núcleo com incorporação de cortiça poderão ser aplicados como alternativa onde houver a necessidade de redução de peso e também onde houver a expectativa de solicitações de flexão superiores às suportadas pela resina pura.

Palavras-chave: Compósito laminado, Cortiça, Microesferas, Flexão.

\begin{abstract}
In this work, epoxy polymer composites laminated with cork and hollow glass microspheres were developed. The laminated composites were manually made and evaluated for the morphological characteristics of the scanning electron microscope and the influence of the incorporation of the fillers was evaluated by means of density and flexural strength. In general, good dispersion of both the cork powder and the hollow glass microspheres was observed in the central layers of the sheet, but the cork-based cores presented better adhesion to the matrix. It was also observed the reduction in the density of all the composites with respect to the pure matrix and that the flexural modulus of cork-based composites and those with hollow microspheres of glass, even with close values, were superior to modulus of bending of the pure epoxy system. Core laminates with cork incorporation may be applied as an alternative where weight reduction is required and also where there is the expectation of bending stresses higher than those supported by the pure resin.
\end{abstract}

Keywords: Laminated Composite, Cork, Microspheres, Flexural.

\section{INTRODUÇÃO}

Os polímeros termorrígidos são uma categoria de materiais que apresentam alto desempenho, durabilidade e segurança sob altas temperaturas, o que os tornam adequados para aplicações especiais que vão desde a indústria automotiva e aeroespacial até embalagens, revestimentos de eletrônicos e produtos de consumo. Os recentes desenvolvimentos em processos de fabricação e tecnologia em termorrígidos ampliaram seu uso exponencialmente nos últimos anos [1].

Com a forte demanda, particularmente do mercado de materiais compósitos, automotivos, 
aeronáuticos/aeroespaciais e indústria eletroeletrônica, o consumo de polímeros termorrígidos mostrou crescimento constante nos últimos anos. Dentre as resinas termorrígidas pode-se destacar a resina epóxi, que teve uma demanda global estimada em aproximadamente US \$ 21,5 bilhões em 2016 [2].

Entre as várias formas de arranjos possíveis em estruturas de compósitos, tornou-se popular a ideia de construção sanduíche devido ao desenvolvimento de materiais de núcleo celulares artificiais fabricados pelo homem, pois apresentam características diferentes das obtidas em materiais sólidos tradicionais [3]. As estruturas sanduíches consistem em um par de camadas rígidas (faces, revestimentos ou capas), um núcleo resiliente e leve para separar as faces e transferir as cargas de uma camada externa para a outra e, por último, um adesivo entre as camadas que seja capaz de transmitir cisalhamento e cargas axiais entre elas [4].

Em termos de carga de núcleo, as microesferas ocas de vidro (MOV) são amplamente utilizadas em materiais compósitos, como espumas sintáticas [5-7]. As microesferas consistem em vidro rígido externo e gás inerte internamente, resultando em algumas propriedades como baixa densidade, baixa condutividade térmica e baixa constante dielétrica. No passado, a maioria das investigações diz respeito à fabricação e propriedades mecânicas de espumas sintáticas para estruturas aeronáuticas e marítimas que requeiram propriedades multifuncionais, incluindo alta resistência à compressão específica, baixa absorção de umidade e estabilidade térmica [8].

Com o avanço em materiais compósitos, polímeros com cargas de origem natural tem despertado grande interesse entre pesquisadores em parte devido à necessidade de se desenvolver materiais sustentáveis, substituindo o petróleo ou subprodutos do vidro que são amplamente usados como reforço nos mesmos [9].

A utilização de reforço de materiais naturais, como a cortiça chamam atenção devido às suas propriedades térmicas (como estabilidade estrutural das células até temperaturas de $250^{\circ} \mathrm{C}$ ), baixa densidade, baixo custo e o seu caráter sustentável como matéria-prima. O uso de materiais lignocelulósicos como enchimento ou reforço em matrizes tem ganhado aceitação em diferentes aplicações pois, semelhantemente à madeira, a cortiça é uma matéria-prima natural, renovável e sustentável com um potencial inexplorado para o desenvolvimento de compostos parcialmente naturais [10-12].

O uso de estruturas leves com boa relação resistência-peso tem sido uma característica procurada na indústria, especialmente de transporte e aeronáutica. A crescente demanda por novos materiais induziu um crescimento significativo na tecnologia de compósitos sanduíche, onde eles são usados para enrijecer aplicações compostas, como cascos de barcos, capôs de automóveis, estruturas de trens e painéis de aeronaves. Os materiais de núcleo comumente usados são colmeias sintéticas, espumas sintáticas e madeira de balsa, mas desenvolvimentos recentes resultaram em novas alternativas, como estruturas de núcleo celular como a cortiça [13].

Segundo WOUTERSON et al. [14] a utilização de microesferas ocas de vidro, que tem uma densidade mais baixa em comparação à maioria das matrizes poliméricas, permite a fabricação de materiais mais leves aumentando-se o volume de carga no compósito. Diferentemente da maioria das outras espumas, a espuma sintática é um material cuja densidade antes é a mesma que após da cura, dado que o volume do interior das microesferas é mantido constante. Essa previsibilidade é vantajosa no processo de fabricação estruturas aeroespaciais, por exemplo.

Com possibilidade de uso das microesferas e outras cargas menos densas em diversas técnicas de produção de compósitos em larga escala, começou-se a encontrar aplicações nas indústrias de transporte, aeroespacial, eletrônica, esporte e lazer. $\mathrm{O}$ uso em transporte pode ser destacado pelo fato da economia média de combustíveis, que segundo o Departamento de Energia dos Estados Unidos, para cada 10\% em peso eliminado em automóveis tem-se 7\% de economia em combustíveis [15].

Segundo WALSH et al. [16] no mundo atual os materiais "verdes" estão se tornando cada vez mais populares na tentativa de economia de energia e redução das emissões de carbono. Com o crescimento do número de estudos examinando o uso de cortiça em compósitos sanduíche, vem sendo demonstrado que a cortiça tem propriedades benéficas de absorção de energia, incluindo a redução de ruído, amortecimento e impacto de baixa velocidade, bem como a redução do peso total em suas aplicações, dada a sua reduzida densidade.

O objetivo deste trabalho é estudar a viabilidade do uso de pó de cortiça como reforço de núcleo de estruturas sanduíche fazendo-se um comparativo com o desempenho de compósitos com incorporação de microesferas ocas de vidro e com amostras de resina pura, avaliando-se a morfologia dos compósitos por Microscopia Eletrônica de Varredura (MEV), estudando-se o efeito das diferentes cargas na densidade dos compósitos e analisando-se o comportamento mecânico dos compósitos sob condições de flexão. 


\section{MATERIAIS E MÉTODOS}

\subsection{Materiais}

No desenvolvimento deste trabalho foi utilizada como matriz polimérica a resina epóxi (EPX), bicomponente, SILAEX SQ 2004, com endurecedor SQ 3154, fornecida pela distribuidora Redelease. Foram utilizadas como cargas a cortiça em pó, fornecida pela Corticeira Paulista LTDA, e microesferas ocas de vidro, provenientes da linha $\mathrm{iM} 16 \mathrm{~K}$, fornecidas pela $3 \mathrm{M}^{\mathrm{TM}}$. As respectivas características granulométricas e de densidade são apresentadas na Tabela 1 .

Tabela 1: Características físicas das cargas utilizadas de acordo com os fabricantes

\begin{tabular}{lc}
\hline CARACTERÍSTICAS & PÓ DE CORTIÇA \\
\hline Densidade $(\mathrm{g} / \mathrm{l})$ & 65 a 85 \\
Mesh $50-(0,30 \mathrm{~mm})(\%)$ & 20 a 50 \\
Mesh $60-(0,25 \mathrm{~mm})(\%)$ & 10 a 45 \\
Mesh $80-(0,18 \mathrm{~mm})(\%)$ & 5 a 30 \\
\hline & \\
CARACTERÍsTICAS & MICROESFERAS DE VIDRO \\
\hline Densidade $\left(\mathrm{g} / \mathrm{cm}^{3}\right)$ & 0,46 \\
Diâmetro médio $(\mu \mathrm{m})$ & 20 \\
Faixa de Tamanho da Partícula $(\mu \mathrm{m})$ & 20 - 29 \\
Resistência ao esmagamento $(\mathrm{MPa})$ & 110,3 \\
\hline
\end{tabular}

Para a confecção dos moldes de silicone foi utilizada a resina bicomponente SILAEX SQ8328 de cura a frio.

\subsection{Preparação dos moldes}

Para a confecção dos corpos de prova foram fabricados moldes de silicone, a partir de amostras de referência com dimensões requeridas pela norma de ensaios de resistência a flexão, ASTM D790. Ao longo de uma base de acrílico foram depositadas amostras de referência e sobre as mesmas foi vertida a resina de silicone para a formação do molde.

\subsection{Formulação e preparação dos compósitos}

Os compósitos laminados foram confeccionados com um total de três camadas: duas camadas externas a base da resina epóxi/endurecedor, na proporção de 100-50, conforme indicado pelo fabricante, e uma camada central de resina epóxi e cargas. Antes de incorporadas à resina, as cargas foram secas a $70^{\circ} \mathrm{C}$ durante $5 \mathrm{~h}$ em estufa para remoção de umidade residual. As frações volumétricas de material no núcleo dos corpos de prova são apresentadas na Tabela 2.

Tabela 2: Frações volumétricas dos materiais constituintes dos núcleos dos compósitos

\begin{tabular}{lccc}
\hline NÚCLEO & EPX & CORTIÇA & MOV \\
\hline Núcleo carga cortiça & $45 \%$ & $55 \%$ & - \\
Núcleo carga MOV & $45 \%$ & - & $55 \%$ \\
\hline
\end{tabular}




\subsubsection{Preparação de núcleos a base de cortiça}

A cortiça foi incorporada à resina epóxi, ainda sem endurecedor, por meio de mistura mecânica em agitador da marca Fisatom, modelo F713D, durante 90 segundos e sob rotação de hélice de 1500 rpm. Após o tempo indicado, o endurecedor foi adicionado à mistura sob agitação permanente durante 90 segundos e em seguida a composição foi vertida sobre o molde de silicone, sendo desmoldada após $72 \mathrm{~h}$, conforme metodologia proposta por BARBOSA et al. [17].

\subsubsection{Preparação dos núcleos a base de Microesferas ocas de vidro}

As MOVs foram incorporadas à matriz de epóxi sob agitação manual durante 90 segundos e posteriormente o endurecedor foi adicionado à mistura permanecendo sob agitação por mais 90 segundos. O objetivo da mistura manual foi evitar a quebra das microesferas que poderia ocorrer caso estas fossem processadas em agitador mecânico. A mistura foi vertida sobre o molde e foi retirada após 72h, após a cura total. O presente procedimento foi baseado no trabalho de PINISETTY et al. [6].

\subsubsection{Formulação e preparação dos compósitos}

Uma vez que todas as camadas passaram pelo processo de cura total, iniciou-se o processo de montagem das estruturas sanduíche, conforme pode ser observado na Figura 1. As camadas foram unidas utilizando-se a mesma resina base como adesivo.

A classificação dos sistemas com relação à composição e arranjo dos materiais deste trabalho pode ser vista na Tabela 3.

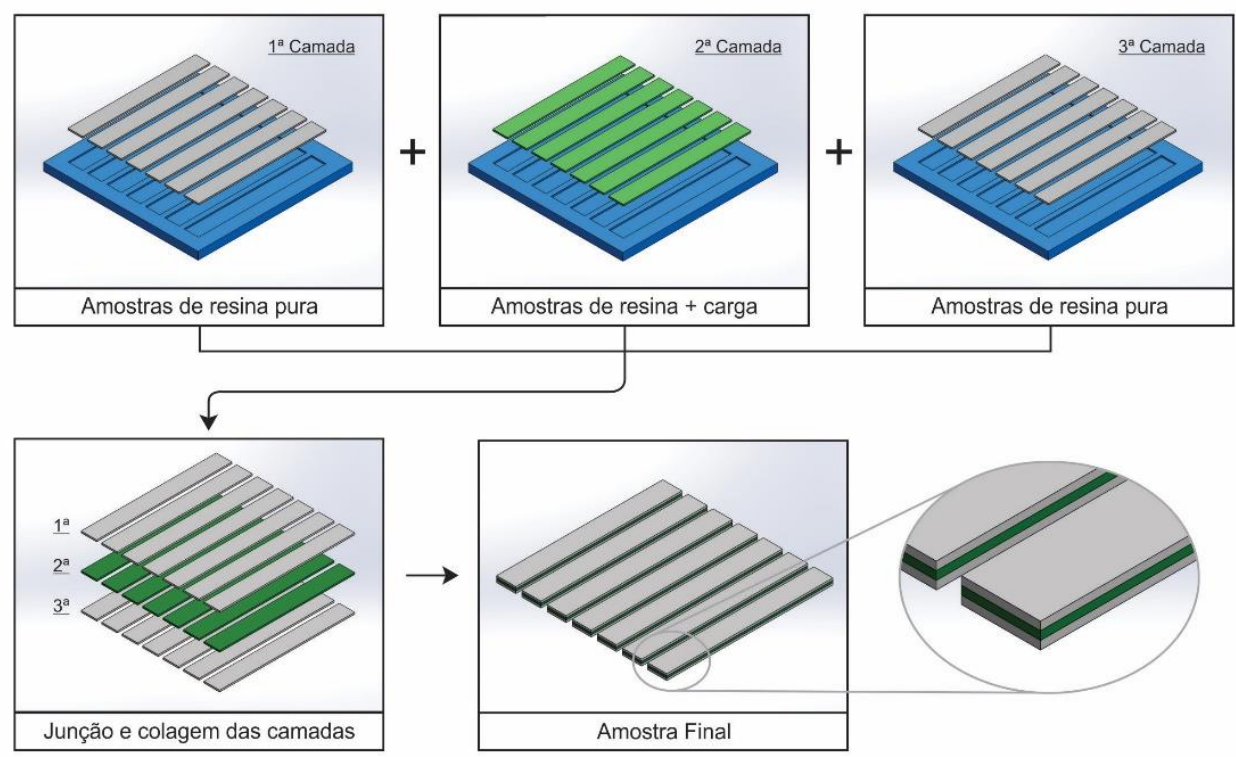

Figura 1: Processo de manufatura dos compósitos

Tabela 3: Formulação dos compósitos

\begin{tabular}{lc}
\hline SIGLA & DESCRIÇÃo \\
\hline EPX & Resina epóxi + endurecedor \\
CC & Compósito sanduíche com núcleo de cortiça \\
CMOV & Compósito sanduíche com núcleo de MOV \\
\hline
\end{tabular}

\subsection{Caracterização morfológica - Microscopia Eletrônica de Varredura}

A morfologia dos compósitos foi analisada por meio de microscopia eletrônica de varredura realizada na superfície de fratura dos corpos de prova, utilizando Microscópio Eletrônico de Varredura Shimadzu Superscan SSX-550 (MEV) sob vácuo e com filamento de tungstênio. A análise foi realizada no Laboratório do Programa de Pós-Graduação em Engenharia de Materiais do Instituto Federal do Piaú - Campus Teresina 
Central. Para a análise da superfície de fratura foi realizada a metalização da amostra por meio da aplicação de uma fina camada de ouro, usando uma corrente de $10-15 \mathrm{~mA}$, no tempo de 2 minutos por amostra. Como parâmetro de comparação, a superfície de fratura da amostra sem carga (EPX) também foi analisada.

\subsection{Densidade}

Para a análise da influência da incorporação das cargas de cortiça e MOV nos núcleos dos compósitos sanduíche, foram realizados ensaios de avaliação de densidade aparente. Foram seguidos os parâmetros de análise propostos na ASTM D70, adaptada, com corpos de prova dos compósitos sanduíches medindo aproximadamente $62,00 \times 3,00 \times 12,50 \mathrm{~mm}$.

Os ensaios foram realizados utilizando-se um picnômetro de $125 \mathrm{ml}$ da marca Astral Científica, pertencente ao Laboratório de Química Analítica, Inorgânica e Físico-Química do Instituto Federal do Piauí Teresina Central. O teste foi executado com seis corpos de prova para cada composição utilizando-se água destilada, monitorando-se a temperatura da mesma com um termômetro de mercúrio. As massas das amostras e do picnômetro foram obtidas por meio uma balança de três algarismos significativos.

\subsection{Caracterização mecânica - ensaio de flexão}

Foi realizado o ensaio de flexão em três pontos de acordo com a norma ASTM D790 em máquina de ensaios universal da marca EMIC, modelo DL 30000N, pertencente ao Laboratório de Ensaios Mecânicos do curso de Engenharia de Materiais da Universidade Federal do Piaú, com uma célula de carga de 50kN a uma taxa de deflexão de $2 \mathrm{~mm} / \mathrm{min}$ e distância total entre os apoios de $5 \mathrm{~cm}$. Avaliou-se o módulo de flexão e força máxima, com 05 amostras ensaiadas para cada sistema.

\section{RESULTADOS E DISCUSSÃO}

\subsection{Caracterização morfológica - Microscopia Eletrônica de Varredura}

Com o intuito de analisar a morfologia dos corpos de prova de resina pura e seus compósitos, foram capturadas imagens por MEV para cada um dos compósitos. A morfologia da resina epóxi e dos compósitos sanduíche na fratura é apresentada na Figura 2. 


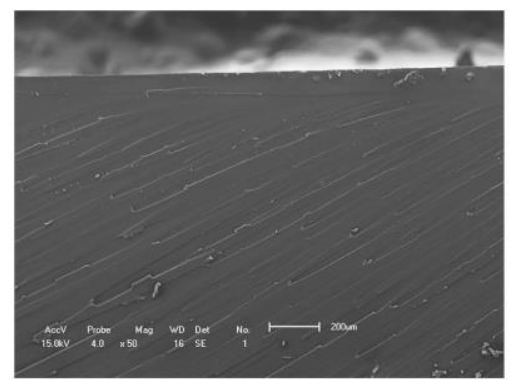

a) $\mathrm{EPX}, 50 \mathrm{x}$

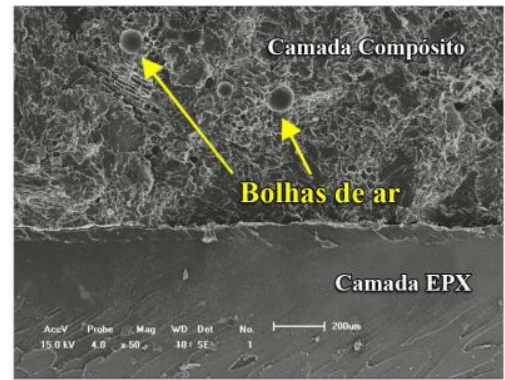

d) $\mathrm{CC}, 50 \mathrm{x}$

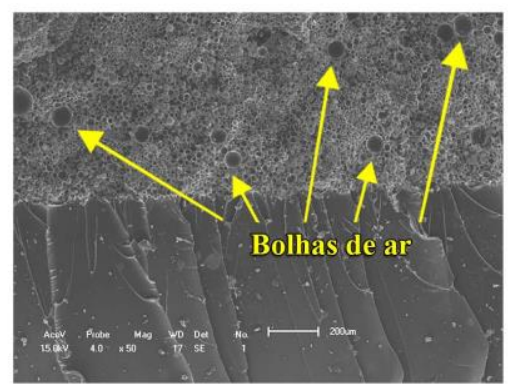

g) $\mathrm{CMOV}, 50 \mathrm{x}$

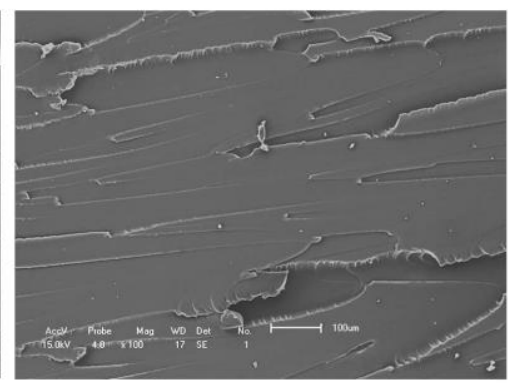

b) EPX, 150x

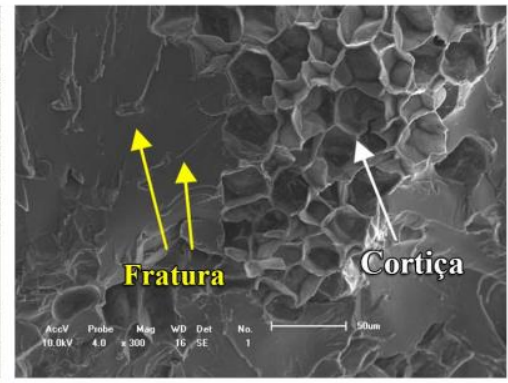

e) $\mathrm{CC}, 300 \mathrm{x}$

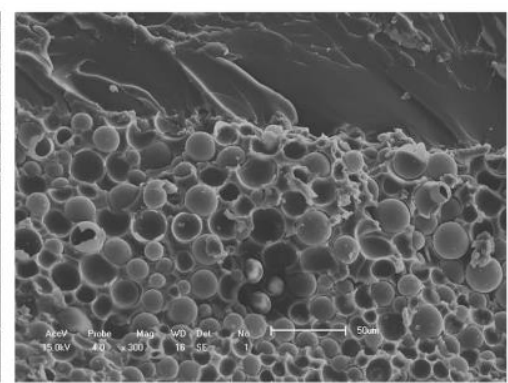

h) $\mathrm{CMOV}, 300 \mathrm{x}$

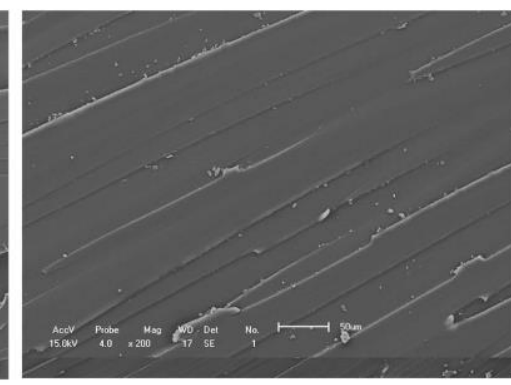

c) EPX, 200x

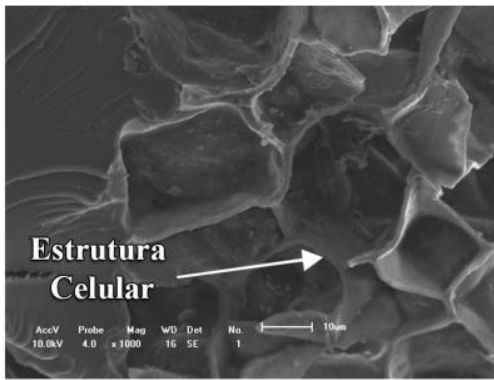

f) $C C, 1000 x$

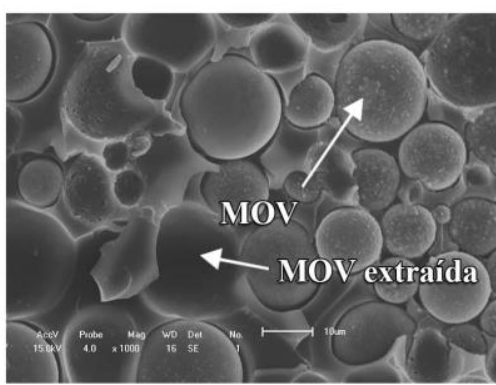

i) $\mathrm{CMOV}, 1000 \mathrm{x}$

Figura 2: Microscopia eletrônica de varredura da superfície de fratura dos corpos de prova

Nas Figuras 2a, 2b e 2c, relativas aos corpos de prova a base de resina epóxi pura (EPX) pode-se observar a fratura frágil inerente ao material, representada pelo perfil da fratura e propagação rápida das trincas, como observado por OPELT et al. [17] em compósito de matriz epoxídica. Conforme dito anteriormente, a resina apresenta características de fratura frágil, por possuir "river partterns" e as marcas propagam-se sofrendo poucos desvios ao longo da matriz polimérica.

Nas amostras dos dois compósitos foram encontradas bolhas de ar que, segundo SHEN et al. [18], são advindas do aumento da viscosidade da mistura resina/carga, o que dificulta a mobilidade das bolhas de ar em direção à superfície.

Nas Figuras 2d, 2e e 2f, referentes ao compósito com cortiça, são exibidas a interação entre as camadas do compósito, a distribuição do pó de cortiça na matriz e a interação da cortiça com a resina. Observa-se a boa adesão entre a camada externa de resina e a camada central do compósito, não sendo observadas ranhuras e nem delaminação após a fratura. BARBOSA et al. [19] ao estudarem compósitos com cortiça observaram que as partículas de cortiça tinham uma estrutura celular semelhante a um favo de mel, compostos por várias células, algumas fechadas (interior da célula intacto) e algumas abertas (nas extremidades da célula) onde há a interação com a matriz, em concordância com o que foi encontrado no presente trabalho. As imagens sugerem ainda que as superfícies das partículas de cortiça aderiram bem à matriz polimérica, uma vez que não foram detectados espaços vazios entre as duas fases.

FERNANDES et al. [20] estudaram o uso de cortiça como reforço de poliésteres, polímeros inerentemente de fratura frágil e ao analisarem as micrografias MEV dos compósitos na região de fratura (após o ensaio de tração) observaram uma superfície com maior rugosidade, com a ausência de vazios ou cavidades, o que denota a transferência de tensão eficiente da matriz à carga de cortiça quando a tensão foi aplicada. 
De forma semelhante, nos corpos de prova CMOV foi observada boa adesão entre a camada externa de resina e o núcleo compósito, não sendo vistas ranhuras e delaminação entre as camadas mesmo após a fratura.

De acordo com a micrografia apresentada nas Figuras $2 \mathrm{~h}$ e $2 \mathrm{i}$ observa-se uma boa dispersão das microesferas na matriz, e, no entanto, houve uma fraca adesão das MOVs à matriz, pois percebem-se vazios entre a carga e o polímero e não há ranhuras se propagando em direção às microesferas, o que indica que a fratura ocorreu ao redor delas. Outra característica dos compósitos CMOV é remoção de microesferas durante o ensaio mecânico. Essa análise corrobora com a conclusão de que houve baixa adesão entre a resina e as microesferas.

Resultados semelhantes foram encontrados por PINISETTY et al. [6] durante a análise de compósitos a base de epóxi com adição de microesferas ocas de vidro de diferentes fabricantes.

KITEY e TIPPUR [21] afirmaram que as ligações fracas entre as partículas e a matriz resultaram em uma resistência inferior na fratura quando comparadas às partículas fortemente ligadas. A força de ligação da matriz/interface de partículas é, portanto, um parâmetro central que determina qual mecanismo de transferência de tensões é dominante no sistema (uma vez que a aderência do enchimento/matriz aumenta a eficiência da transferência).

\subsection{Densidade}

Para a análise da influência da incorporação das cargas nos sistemas CC e CMOV, foram realizados ensaios de determinação de densidade aparente. Os ensaios foram executados com água destilada sob temperatura ambiente registrada em $27^{\circ} \mathrm{C}$. As médias dos resultados encontrados são apresentadas na Tabela 4.

Tabela 4: Densidade dos compósitos

\begin{tabular}{lc}
\hline SISTEMAS & DENSIDADE APARENTE MÉDIA $\left(\mathbf{g} / \mathbf{c m}^{3}\right)$ \\
\hline EPX & $1,249 \pm 0,036$ \\
CC & $1,161 \pm 0,022$ \\
CMOV & $1,123 \pm 0,032$ \\
\hline
\end{tabular}

Os resultados da Tabela 3 apontam uma redução de densidade nos sistemas CC e CMOV com relação ao sistema EPX de, respectivamente, 7,0 e 10,0\%. Esse resultado já era esperado devido às características das cargas que apresentavam uma densidade inferior à da matriz polimérica. A tendência da redução pode ser explicada pela introdução de preenchimento de vazios internos por nanopartículas com densidade aparente $(0,011 \mathrm{~g} / \mathrm{cm} 3)$ inferior à densidade da própria resina $(1,13 \mathrm{~g} / \mathrm{cm} 3)$.

\subsection{Caracterização mecânica - Ensaio de Flexão}

Foram realizados ensaios de resistência à flexão em três pontos para todos os sistemas e os respectivos resultados são apresentados na Tabela 5.

Tabela 5: Resultados do ensaio de flexão dos compósitos.

\begin{tabular}{lll}
\hline SISTEMAS & MÓDULO DE FLEXÃO $(\mathrm{MPa})$ & TENSÃO DE FLEXÃO $(\mathrm{MPa})$ \\
\hline EPX & $512,51 \pm 26,22$ & $13,77 \pm 1,49$ \\
CC & $695,32 \pm 25,63$ & $20,46 \pm 1,20$ \\
CMOV & $718,72 \pm 50,9$ & $19,88 \pm 1,78$ \\
\hline
\end{tabular}

Os resultados para o módulo de flexão e força máxima são apresentados respectivamente nas Figuras 3 e 4 e de uma maneira geral, foi possível observar o aumento da rigidez dos compósitos quando comparados à matriz pura, sendo o maior percentual obtido na presença de microesferas de vidro. Esse comportamento pode ser explicado pela característica diferencial das MOVs de alta eficiência sob compressão [22,23].

Segundo SCHULTE et al. [24] esses resultados podem ser atribuídos em parte aos enchimentos que absorvem as tensões na interface carga-epóxi e também devido às tensões cisalhantes presentes na interface entre as camadas, que reduzem a mobilidade e a consequente capacidade de se deformar nos compósitos.

Em polímeros reforçados por partículas, é comum observar a resistência à flexão sendo superior à 
resistência sob tração. Sob uma situação de carga de flexão, em vez de atuar como concentradores de tensão, como acontece no carregamento por tração, as cargas aparentemente auxiliam na capacidade de transferência interna de tensões no compósito. Segundo SUDHEER et al. [25] isto se deve ao fato de que o esforço compressivo tende a fechar rachaduras e falhas que são perpendiculares à tensão aplicada.

Comparando as cargas, as microesferas de vidro têm como característica inerente a alta resistência à compressão, o que consequentemente promoveu um aumento no desempenho dos compósitos. Entretanto, a cortiça apresenta um desempenho ligeiramente superior às microesferas de vidro em função da absorção de energia permanecendo íntegra quanto à sua estrutura aveolar o que tende a explicar as maiores forças alcançadas neste sistema (BARBOSA et al. [26].

De acordo com ZHENG et al. [27] o material sob flexão fica submetido a forças envolvendo tensões de cisalhamento na região de compressão do compósito laminado. Por sua vez, CAO e CAMERON [28] argumentam que o ganho em resistência à flexão é devido ao aumento de tensões cisalhantes no laminado, pois esses dividem as tensões por toda superfície da partícula ou fibra, justificando incremento de força.

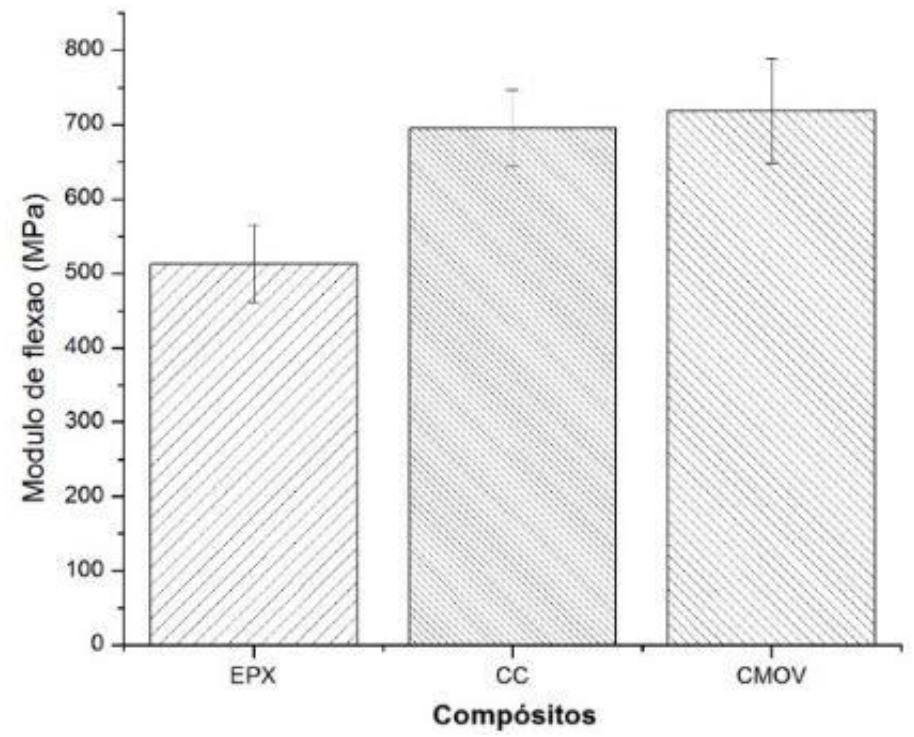

Figura 3: Módulo de flexão dos compósitos

Chung [29] afirma que as propriedades de resistência do material na flexão possuem relação direta com a região de interface. Com uma região de interface fraca, a transferência de carga entre matriz e fibra é reduzida, limitando a resistência à flexão do material apenas pela fase matriz. A matriz flete e como a tenacidade da partícula é maior, ocorre uma maior absorção de energia [30] e o material sofre a falha quando a matriz chega ao seu limite. Corroborando com os resultados de MEV, houve a formação de interface homogênea entre as cargas e a matriz epóxi e que dentro das condições analisadas, mesmo sem a presença de um agente compatibilizante, houve o aumento das propriedades mecânicas dos compósitos. 


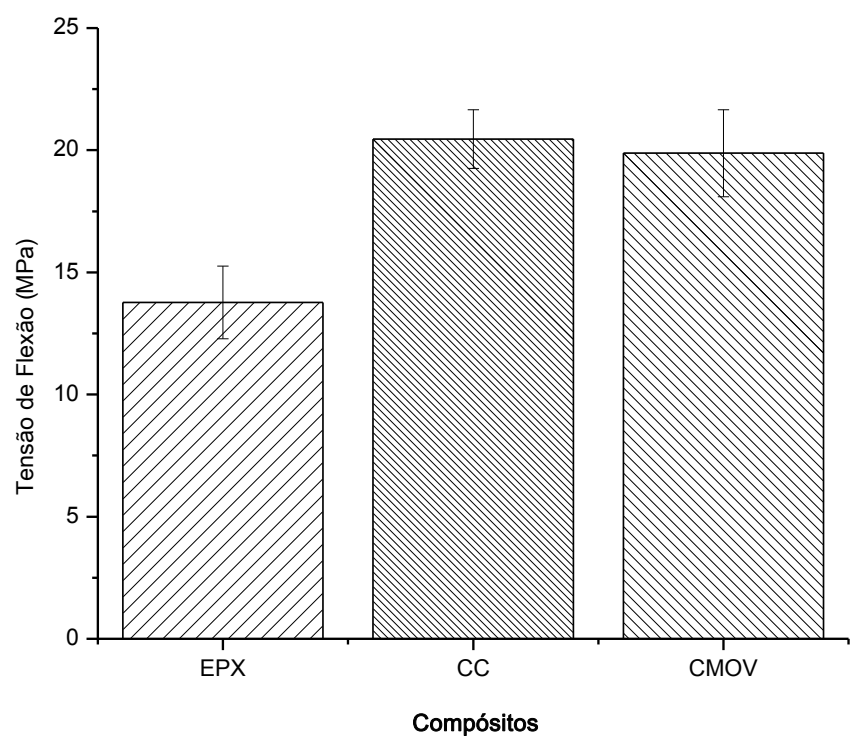

Figura 4: Tensão de flexão dos compósitos

Observou-se maiores valores para tensão de flexão (Figura 4) para os compósitos laminados com relação ao sistema EPX, aumento este em média de $41 \%$ do sistema CC e de $27 \%$ do sistema CMOV com relação à resina pura.

Com relação a compósitos com CMOV, GUPTA et al. [23] em seus estudos do uso de espumas sintáticas afirmaram que uma característica típica é à absorção de energia das microesferas no processo de compressão.

Durante o processo de esmagamento, as partículas de vidro oco tendem a fraturar e expor o volume fechado por elas. Assim, no sistema CMOV supõe-se que haja a fratura de algumas das microesferas na região superior do compósito onde predominam forças compressivas, dissipando a energia sob flexão no mecanismo de fratura das MOVs. Segundo WOUTERSON et al. [14] ainda há o efeito da curvatura da propagação das trincas ao redor das microesferas no estado de compressão, o que promove o enrijecimento do material. Neste tipo de estruturas, quando submetidas à flexão, as faces são responsáveis por suportar os esforços axiais resultantes (tração e compressão), enquanto que o núcleo suporta os esforços de corte gerados pela tendência do deslizamento entre as duas peles.

De acordo com os resultados apresentados, percebeu-se que mesmo sendo cargas de núcleo diferentes, as cargas de cortiça e microesferas ocas de vidro compartilharam algumas semelhanças (presença de ar em suas células, ar este que não entra em contato com a matriz a não ser que a célula seja rompida).

Segundo Pethrick et al [31], matrizes epóxi termorrígidas normalmente têm uma baixa resistência à propagação de trincas, e através da incorporação de uma segunda fase particulada seja ela em escala micro ou nano ou mesmo a combinação das duas pode-se aumentar a resistência mecânica final do compósito. Entretanto, sabe-se que para lidar com as limitações dos polímeros, como sua baixa rigidez e baixa resistência mecânica, partículas inorgânicas podem ser adicionados para aumento da rigidez do polímero [3234]. 


\section{CONCLUSÕES}

Compósitos laminados a base de resina epóxi, cortiça e microesferas de vidro ocas foram preparados e avaliados quanto à morfologia, densidade e resistência mecânica à flexão. De acordo com os resultados obtidos, pode-se concluir que houve uma boa dispersão das cargas, mesmo com processamento manual, e que a inserção das mesmas promoveu a redução da densidade dos compósitos em níveis de $7 \%$ na presença de cortiça e $11 \%$ e microesferas de vidro oca. Mecanicamente, o módulo de flexão e a tensão de flexão foram superiores a matriz polimérica, apresentando valores próximos entre as diferentes cargas, indicando que, mesmo com morfologias diferentes, uma pode ser substituta da outra a depender da aplicação.

\section{AGRADECIMENTOS}

Ao Programa de Pós-Graduação em Engenharia de Materiais do Instituto Federal do Piauí - PPGEM (IFPI), ao Laboratório Interdisciplinar de Materiais Avançados - LIMAV (UFPI) e ao Laboratório de Polímeros e Materiais conjugados - LAPCON (UFPI) pelo espaço cedido para o desenvolvimento dos compósitos e equipamentos necessários para a realização das caracterizações. À CAPES e ao CNPq pelo apoio financeiro.

\section{BIBLIOGRAFIA}

[1] GOODMAN, S. H., HANNA, D., Handbook of thermoset plastics. William Andrew, 2014.

[2] GLOBAL EPOXY RESIN MARKET, Acmite Market Intelligence, p. 575, 2017.

[3] PETRAS, A., Design of sandwich structures, University of Cambridge, 1999.

[4] ASTM C274-07, Standard Terminology of Structural Sandwich Constructions, West Conshohocken, PA: ASTM International, 2012.

[5] GUPTA, N., PINISETTY, D., SHUNMUGASAMY, V. C., Reinforced polymer matrix syntactic foams: effect of nano and micro-scale reinforcement, Springer Science \& Business Media, 2013.

[6] PINISETTY, D., SHUNMUGASAMY, V. C., GUPTA, N., "Hollow Glass Microspheres in ThermosetsEpoxy Syntactic Foams", In: Hollow Glass Microspheres for Plastics, Elastomers, and Adhesives Compounds pp.147-174, 2015

[7] GUPTA, N., NAGORNY, R., "Tensile properties of glass microballoon- epoxy resin syntactic foams", $J$. Appl. Polym. Sci., v. 102, n. 2, pp. 1254-1261, 2006.

[8] PARK, S.-J., JIN, F.-L., LEE, C., "Preparation and physical properties of hollow glass microspheresreinforced epoxy matrix resins", Mater. Sci. Eng. A, v. 402, n. 1, pp. 335-340, 2005.

[9] YEO, J., KIM, O. Y., HWANG, S., "The effect of chemical surface treatment on the fracture toughness of microfibrillated cellulose reinforced epoxy composites", J. Ind. Eng. Chem., p. 24, 2016.

[10] AROSO, I.M., FERNANDES, E.M., PIRES, R.A., et al., "Cork extractives exhibit thermo-oxidative protection properties in polypropylene-cork composites and as direct additives for polypropylene", Polym. Degrad. Stab., v. 116, pp. 45-52, Jun. 2015.

[11] FERNANDES, E.M., CORRELO, V.M., CHAGAS, J.A.M., et al., "Properties of new cork-polymer composites: Advantages and drawbacks as compared with commercially available fibreboard materials", Compos. Struct., v. 93, n. 12, pp. 3120-3129, 2011.

[12] FORTES, M., PEREIRA, H., ROSA, M., Cortiça, 2nd ed. Lisboa, IST Press, 2004.

[13] VINSON, J. R., SIERAKOWSKI, R. L., The behavior of structures composed of composite materials, v. 5, Springer Science \& Business Media, 2012.

[14] WOUTERSON, E. M., BOEY, F.Y.C., HU, X., et al., "Specific properties and fracture toughness of syntactic foam: Effect of foam microstructures", Compos. Sci. Technol., v. 65, n. 11, pp. 1840-1850, 2005.

[15] AMOS, S.E., YALCIN, B., Hollow Glass Microspheres for Plastics, Elastomers, and Adhesives Compounds. Chadds Ford, PA, Elsevier, 2015.

[16] WALSH, J., KIM, H. I., SUHR, J., "Low velocity impact resistance and energy absorption of environmentally friendly expanded cork core-carbon fiber sandwich composites", Compos. Part A Appl. Sci. Manuf., v. 101, pp. 290-296, 2017. 
[17] OPELT, C. V, CONCEI, K., COELHO, L. A. F., "Tenacidade à fratura de resina epóxi DGEBA com incorporação de copolímero em bloco (PEO-PPO-PEO)e Grafeno", In: Congresso Brasileiro de Engenharia e Ciência dos Materiais Cuiabá, MT, Brasil, pp. 7684-7691, 2014.

[18] SHEN, J., HUANG, W., WU, L., et al., "The reinforcement role of different amino-functionalized multiwalled carbon nanotubes in epoxy nanocomposites", Compos. Sci. Technol., v. 67, n. 15-16, pp. 3041-3050, 2007.

[19] BARBOSA, A.Q., SILVA, L.F.M.F.M., ÖCHSNER, A., et al., "Utilização de micro partículas de cortiça como material de reforço em adesivos estruturais frágeis", Cienc. e Tecnol. dos Mat., v. 25, n. 1, pp. 4249, 2013.

[20] FERNANDES, E.M., CORRELO, V.M., MANO, J.F., et al., "Cork-polymer biocomposites: Mechanical, structural and thermal properties", Mater. Des., v. 82, pp. 282-289, 2015.

[21] KITEY R., TIPPUR, H.V., "Role of particle size and filler-matrix adhesion on dynamic fracture of glass-filled epoxy. I. Macromeasurements", Acta Mater., v. 53, n. 4, pp. 1153-1165, 2005.

[22] GUPTA, N., ZELTMANN, S.E, SHUNMUGASAMY, V. C., et al., "Applications of polymer matrix syntactic foams", Jom, v. 66, n. 2, pp. 245-254, 2014.

[23] GUPTA, N. "Response of Syntactic Foam Core Sandwich Structured Composites to Three-Point Bending”, J. Sandw. Struct. Mater., v. 4, n. 3, pp. 249-272, 2002.

[24] SCHULTE, K. et al., Polymer Composites from Nano-to Macro-Scale, New York, NY, 2005.

[25] SUDHEER, M., PRABHU, R., RAJU, K., et al., "Effect of Filler Content on the Performance of Epoxy / PTW Composites", Adv. Mater. Sci. Eng., v. 2014, pp. 1-11, Apr. 2014.

[26] BARBOSA, A.Q., DA SILVA, L.F.M.F.M., ÖCHSNER, A., et al., "Influence of the Size and Amount of Cork Particles on the Impact Toughness of a Structural Adhesive", J. Adhes., v. 88, n. 4, pp. 452-470, 2012.

[27] ZHENG, Y., NING, R., ZHENG, Y. "Study of $\mathrm{SiO}_{2}$ Nanoparticles on the Improved Performance of Epoxy and Fiber Composites", Journal of Reinforced Plastics and Composites, v.24, n.3, pp. 223-233, 2005.

[28] CAMERON, Y.C., "Flexural and shear Properties of Silica Particle Modified Glass Fiber Reinforced Epoxy Composite", Journal of Reinforced Plastics and Composites, v.25, pp. 347-359, Aug.2006.

[29] CHUNG, D. D. L. Carbon Fiber Composites, Butterworth-Heinemann, Boston, 1994.

[30] VENTURA, A. M. F.M. "Os Compósitos e a sua aplicação na Reabilitação de Estruturas metálicas", Ciência e Tecnologia dos Materiais, v. 21, n. 3-4, pp. 10-19, 2009.

[31] PETHRICK, R. A., MILLER, C., RHONEY, I. "Influence of nanosilica particles on the cure and physical properties of an epoxy thermoset resin", Wiley Interscience, v.59, pp. 236-241, Dec.2009.

[32] FU, S.-Y., FENG, X.-Q, LAUKE, B., et al., "Effects of particle size, particle/matrix interface adhesion and particle loading on mechanical properties of particulate-polymer composites", Composites Part B: Engineering, v. 39, n. 6, pp. 933-961, Set. 2008.

[33] QUARESIMIN, M., VARLEY, R. J. "Understanding the effect of nano-modifier addition upon the properties of fibre reinforced laminates", Composites Science and Technology, v.68, n.3-4, pp. 718-726, Mar, 2007.

[34] SANTOS, J. C., Compósitos laminados têxteis de vidro e carbono em matriz epóxi reforçada com micro e nano sílica, Dissertação de M.Sc., UFSJ, São João Del Rei, MG, Brasil, 2013.

\section{ORCID}

Bruno Santos Pereira

Tatianny Soares Alves

Renata Barbosa
https://orcid.org/0000-0002-8960-215X

http://orcid.org/0000-0002-6790-3314

http://orcid.org/0000-0001-6969-394X 